\title{
An Efficient Scheme for MANET Domain Formation (ESMDF)
}

\author{
Adwan AbdelFattah \\ Computer Science Department, \\ The Arab American University of Jenin \\ Jenin, Palestine
}

\begin{abstract}
Mobile Ad hoc Network (MANET) has random topology as MANET devices leaving or joining to the network at anytime. The dynamic nature of MANETs makes achieving secrecy, connectivity and high performance, a big challenge and a complex task. In this paper, we proposed an efficient technique for Dynamic construction of large MANET based on division the network into interoperable domains. This technique is a hybrid of centralized and distributed control of packets forwarding that balances power consumption, minimizes the routing tables and improves the security features. The principles of domain formation based on joining adjacent devices into one group which controlled by one capable device called domain controller. The presented scheme enhances the throughput and the stability of large MANET by minimizing the flooding of messages for keeping track of Devices and during the domain formation.
\end{abstract}

Keywords- MANET; ESMDF; Domain; Domain Router; Domain Formation.

\section{INTRODUCTION}

Mobile Ad-hoc Network (MANET) is a wireless network with dynamic topology. In MANET each node is free to move randomly, and is considered to be equal to other nodes. Each node is capable of transferring the data between the arbitrary source and destination. Thus, each node in MANET can act as a source or destination or router [1].

MANETs are in areas where rapid deployment and dynamic reconfiguration are necessary and wired network is not available. These include military battlefields, emergency search, rescue sites, classrooms and conventions, where participants share information dynamically using their mobile devices.

MANET routing and topology management has become an important issue. Many efficient routing protocols have been developed which ensure the connection of sending and receiving nodes with minimum delay and unnecessary control overhead. Existing routing protocols for MANET can be classified into four different basic categories namely flooding, proactive routing, reactive routing and dynamic cluster based routing [2]. However none of these routing schemes guarantees constant network connectivity during the movement and each of these schemes has constant route maintenance overhead. A particular node may even be disconnected in the worst case. Centralized topology management schemes [3, 4, and 5] discuss a self-adaptive movement control algorithm, which ensures the retention of network connectivity even during the nodes movement. But in this case, the coordinator has to be elected and all other nodes should follow the instructions from the coordinator. The main disadvantages of the centralized topology management scheme are increase in control overhead and non-scalability.

Distributed topology management schemes [6, 7] are generally scalable and adaptive to mobility due to the fact that each node relies on local information collected from nearby nodes. The information obtained by each node is limited, and the strong connectivity of the node is not achieved in this approach.

MANET's characteristics create challenges in several areas. The hosts in the MANET have a limited battery power. In the case of large MANET's, a flat structure may not be the most efficient organization for routing between nodes. Instead, many clustering schemes have been proposed that organize the MANET into a hierarchy, with a view to improve the efficiency of routing. It is important that cluster formation and maintenance should not be costly, in terms of resources used such as bandwidth, battery power etc. Otherwise, the purpose of clustering is defeated.

\section{RELATED WORKS}

In this section, we describe some of the most important protocols and clustering schemes that have been proposed to enhance the quality of service (QoS) and many features of MANETs.

R. Braden, D. Clark, and S. Shenker [8] proposed Integrated Services (IntServ protocol which provides a high level of assurance in fixed network, limited QoS support for mobile networks infrastructure. It requires a high processing power, and this protocol does not support fast QoS changes.

Yu-Xuan Wang[9] proposed an entropy-based WCA (EWCA) which can enhance the stability of the Network for the high mobility of nodes but this leads to high frequency of rejoining which will increase the network overhead. The authors discussed that in the revised algorithm (EWCA-TS) in which performance has been improved with respect to the original WCA, especially on the number of clusters and the rejoining frequency. 
S. Blake, et al [10] proposed Differentiated Services (DiffServ) Protocol which can be easily implemented with MANET but has low level of assurance. It does not guarantee service on end to end basis.

J. Wu and $\mathrm{H}$. L. Li [11] proposed scheme, for routing based on a set of dominating nodes which function as the cluster heads and relay routing information and data packets. The vertices of a Dominating Set (DS) act as cluster heads and each node in a MANET is assigned to one cluster head that dominates it. A DS is called a Connected Dominating Set (CDS) if all the dominating nodes are directly connected to each other.

Qi Xue and Aura Ganz [12] Ad-Hoc QoS On-demand Routing(AQOR) protocol AQOR deals with bandwidth and end to end delay. This protocol includes three main steps: on demand route discovery, signaling function and hop to hop routing. This protocol does not deal with the latency delay.

Taewook Kang, et al [13] proposed a new method for selecting cluster heads to evenly distribute cluster heads and they show that their scheme reduces energy dissipation and prolongs network lifetime as compared with others. They tried to evenly distribute cluster heads over the whole network and avoid creating redundant cluster heads within a small range so that it can increase the network lifetime.

M. Mirhakkak, et al [14] proposed Dynamic Source Routing RSVP (DSRRSVP) protocol which can be easily implemented with DSR routing, but this protocol applicable to a small network with low mobility.

Y.Z.P Chen and A.L Liestman [15] proposed Weakly Connected Dominating Set (WCDS) which relaxed some of the rules of Wu's Algorithm to form a Weakly Connected Dominating Set. There are many disadvantages with the CDS algorithm. The cluster head in CDS algorithm dissipates more power as compared to other nodes in the cluster since all intercluster routing and forwarding happen through it alone. Hence it has a shorter lifespan than the other nodes in the cluster. The cluster head re-election is done after the cluster head dies or moves out of the range of the cluster. This re-clustering incurs a large communication overhead and power dissipation.

Hannan XIAO, et al [16] proposed Flexible QoS Model for MANET(FQMM). FQMM is the first QoS model for MANET. This model is hybrid of both IntServ and DiffServ. Problems of DiffServ and IntServ are present.

Tzay-Farn Shih And Hsu-Chun Yen [17] developed A Location-Aided Cluster-Based Routing Protocol Called Core Location-Aided Cluster-Based Routing Protocol (CLACR). They show that CLACR can be extended as a Geo-Casting Routing Protocol easily, the location Server and Cluster Head can provide location services for different applications. The performance of their routing protocol is better than other protocols.

SWAN Project [18] Service Differentiation in Wireless Ad-hoc Network (SWAN) protocol which uses rate control of TCP and UDP traffic to maintain manageable levels of congestion in the network. It uses admission control for real time traffic and varies the rate of TCP traffic based on feedback from MAC layer to maintain delay and bandwidth bounds for real time traffic. The throughput of this protocol is very low. This protocol does not scale well with high mobility.

Vikas Kawadia and P.R.Kumar [19] proposed CLUSTERPOW algorithm in which dynamic and implicit clustering is done on the basis of transmit power level. The transmit power level is the power level required to transmit each packet. The transmit power level to a node inside the cluster is the less as compared to the level required to send a node outside the cluster. So here the clustering is done keeping the nodes with lower transmit power level together. The primary drawback of their scheme is that there is no cluster head or cluster gateway. Each node here has routing tables corresponding to different transmit power levels. The routing table for a power level in a node is built by communicating with the peer routing table of the same power level at another node. The next hop to route the packet is determined by consulting the lowest power routing table through which the destination is reachable.This approach suggests that each node should know the route to other nodes and also know the transmission power level at which a destination node is reachable. This leads to the overhead of collecting the power level state information and building many routing tables in each node.

P.Basu, et al [20.] proposed clustering scheme which takes aggregate of local mobility as the metric for cluster formation. In such schemes, a cluster is formed by grouping mobile nodes moving with the same velocity. Each node broadcasts two hello packets, separated by a time interval, to its neighbors.

Every node calculates the relative mobility for each of its neighbors using the signal strength of the hello packets received from each adjacent node. Each node then calculates its aggregate mobility as the average of the relative mobility of its neighbors and broadcasts it to the other nodes. The node with the lowest aggregate mobility is chosen as the cluster head. This requires larger communication overhead and a higher latency in cluster formation.

\section{Proposed Domain Formation SCHEME}

We define the following terminologies that are used in the remaining sections.

Domain: Is a set of related devices that can be connected directly or through Domain Router.

Domain Router (DR): Domain Server that performs the following functions:

- Routing the messages among its clients.

- Resolve the problem of Domains merging.

- Forwards the messages to neighbor domains.

- Resolve the IP conflict.

- Limiting the Domain boundaries.

Co-Domain Router (CDR): it is the nearest device to DR, it is selected by DR. CDR should be capable of performing the same function as DR. CDR used to enhance the stability of MANET topology and to minimize the domain formation processes messages. 
Inter Domain Router (IDR): A gateway among domains, this device can forward messages to the neighboring DRs and their clients.

Client Router (CR): A Domain member.

Client fringe (CF): A device that has only one connection with CR, its domain id (DI) $=\mathbf{0}$.

\section{A. Domain properties}

Each domain consists of two or more devices and each individual device interacts directly with other devices in a peer-to-peer fashion. At any time each device can be only in one state (DR, CDR, IDR CR or CF). The state of device is determined by its capability and its location. See fig. 1.

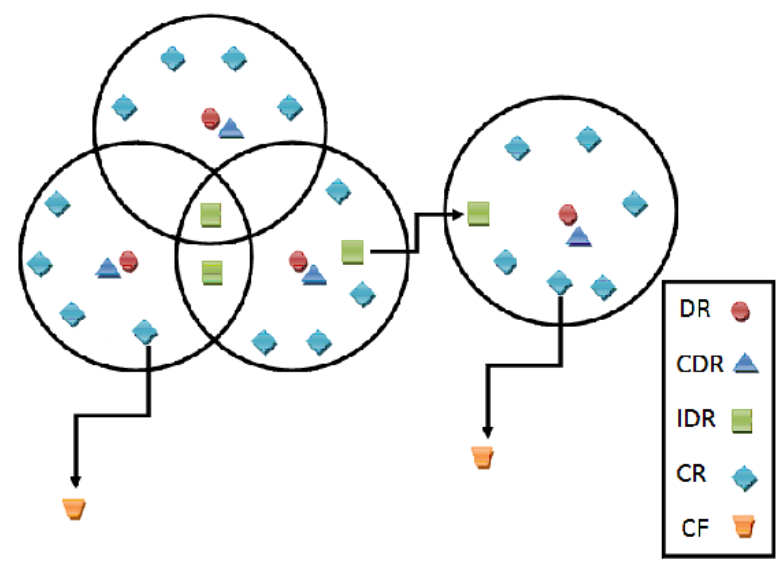

Figure1. Domains representation in MANET

The device mobility changes the domains structure of MANET and consequently the state of devices. Fig.2 describes the devices transition state diagram .

\section{B. Improving Domain Properties}

The proposed scheme of domain formation satisfies the goal of building energy conserving and adaptable domains. This scheme tries to distribute the responsibility among the individual entities. No single entity is in charge of the overall organization. In our proposed scheme, joining new nodes to the domain can be achieved smoothly because of obtaining the information directly from DR or CR.

In our scheme each DR has a CDR that can operates as DR in case of unavailability or over load of DR, and this approach enhances connectivity and decreases power consumption in DR. The process of messages forwarding is very easy, it doesn't need to maintain a huge routing table, as each device store a table of addresses of its neighbors. Each DR can communicate with one or more IDR which is very useful in balancing the load of IDR and in maintaining the connection among domains in case of unavailability of one IDR.

We assume that during device movements there is no far jumps from one domain to the remote one and this mean that only adjacent domains can be affected by these movements which confines the flooding of state change messages and routing information among them.

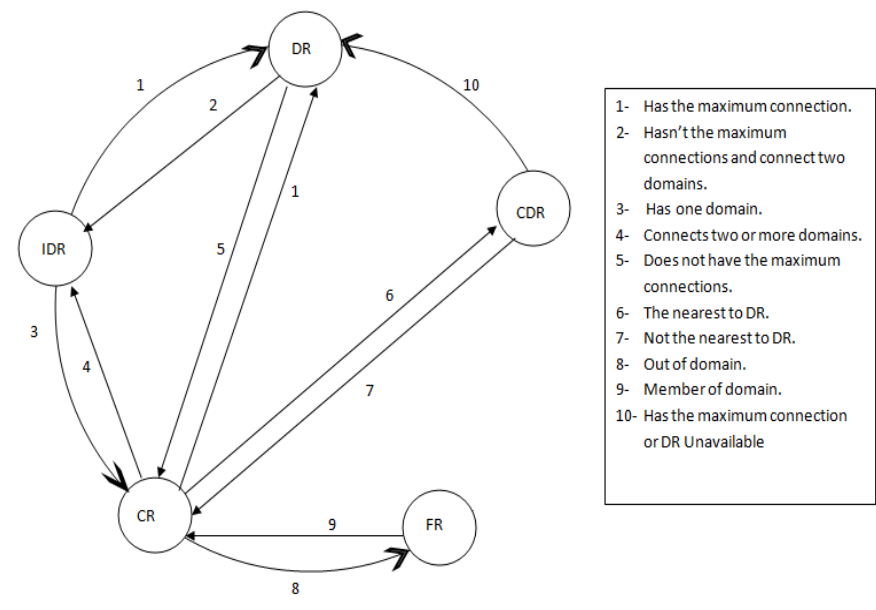

Figure2. Transition state diagram of MANET Devices

\section{DR and CR selection}

The selection criteria of DR is an important issue as it affects over all network .generally there are many aspects that affect the selection of DR as Power level(P), connectivity(C) and Mobility(M).

Generally the DR is selected based on any one of the above mentioned factors, but there are some approaches that considered all of these factors, by getting summation of $\mathbf{P}, \mathbf{C}$ and $\mathbf{M}$, and the selection of DR based on the greatest sum.

In our work we didn't consider the mobility factor as it is difficult to determine and specially at the begging of device state determination process as this factor is unpredictable because of its dynamic characteristics which leads to incorrect DR selection or frequent DR reformation which increase network overhead.

The proposed work considers the following factors:

- Power level $(\mathbf{P})$-The remaining power in the battery of Mobile device, and this factor changed during the running process.

- The Ability (A)-The ability of device depends on many factors: Processor Power, Bandwidth and the installed systems. This factor is predefined and doesn't change during the transaction process with others. This factor contains logical value (True or false).

- Connectivity $(\mathbf{C})$ - Number of connected devices, the maximum $\mathbf{C}$, the maximum Connectivity.

The first two factors are very important and we couldn't include them in a quantitative calculation as the absences of one of them will violate the role of these devices. Instead we replace factors $\mathrm{P}$ and $\mathrm{A}$ by one parameter which we will call it Readiness factor $(\mathbf{R})$.

This factor mean that the device is capable of being DR, IDR or CDR. The value of $\mathbf{R}$ can be false or true and simply computed by using algorithm 1 .

DR selection consists of two phases: 


\section{1) Domain refinement.}

At this step the devices that are not capable to be DR

Will be excluded from DR candidates list and this can be accomplished by using algorithm 1

Algorithm1. Finding the Readiness factor(R) of device

//AP- Acceptable Power level

//MP- maximum power

$\mathrm{AP}=\mathrm{MP} / 2$;

$\mathrm{R}=$ false

If $\mathrm{A}=$ true then

Begin

Repeat

If $\mathrm{P}>=\mathrm{AP}$ then $\mathrm{R}=$ true

Else $\mathrm{AP}=\mathrm{AP}-$ 0.1.AP

Until $\mathrm{R}=$ True or $\mathrm{AP}<0.1$.MP or Ready message received

If $\mathrm{R}=$ true Send ready message.

End.

\section{2) DR Determination}

After determination of $\mathrm{R}$, the process of $\mathbf{D R}$ selection became very simple and can be described by the following algorithm2.

\section{Algorithm2. DR Selection}

- Each device sends Hello Message (HM) that informs neighbors about its existence. Then starts to compute $\mathbf{R}$ by using algorithm1. At the end of this step each Device maintain a list of neighbors, and DR candidates.

- Each capable Device( $\mathrm{R}=$ true) Multicast the number of connected devices(C) to DR candidates.

- $\quad \mathrm{DR}$ is the device with Maximum $\mathrm{C}$, and changes its current state to DR State.

- Wining DR Broadcasts a message which we call DR Wining Message (DRWM) to inform all domain members.

- Domain members change their state to $\mathrm{CR}$ and send Change state Message (CSM) to the neighbors, this step prevents inclusion of $\mathrm{CR}$ into more than one Domain and help the others members to correct their C factor.

The states are arranged into a priority manner to enhance the state selection criteria of each member which based on the capability, number of connections and location. The state priority of DR is the highest one as shown in table1.

TABLE1. State Priority of Domain Members.

\begin{tabular}{|c|r|r|r|r|l|}
\hline Domain Member & \multicolumn{1}{|l|}{ DR } & IDR & CDR & CR & CF \\
\hline Priority & 1 & 2 & 3 & 4 & 5 \\
\hline
\end{tabular}

C. IDR Selection

The selection of IDR consists of two phases:
The first phase is finding the readiness factor $(\mathbf{R})$ of the device by using algorithm1, and the second phase can be described in algorithm 3 .

Algorithm3. IDR Determination

// R[CR i]- $\mathrm{R}$ factor of $\mathrm{i}^{\text {th }} \mathrm{CR}$.

// C- number of Client Routers.

For $\mathrm{i}=1$ to $\mathrm{C}$

Begin

If $(\mathrm{R}[\mathrm{CRi}]=$ true ) and ( $\mathrm{CRi}$ connected to another(DR(s)or $\mathrm{CR}(\mathrm{s})$ or IDR(s) ) from different domain ) Then

Begin

Inform all available DR

If CR received an acceptance message then

CRi changes its state to IDR

End

End.

Before changing CRs state to IDR state it has to receive Acceptance message from all available DRs to enhance security measures and maintenance issues

\section{CDR Selection}

The selection of CDR is initiated by DR by the following conditions:

- CDR should pass readiness factor test.

- $\quad$ CDR is the nearest CR to DR.

The first condition can be achieved by using algorithm1, and the second condition can be accomplished by using algorithm4.

Algorithm4. Determination the Nearest CR

//CDRTP- CDR Transmission Power

//DRTP- DR Transmission Power

// MXTP- Maximum Transmission Power

// MNTP-Minimum Transmission Power

$\Omega=0.05$.MXTP

$\Delta=$ MNTP

CDRFound=false

Repeat

Send CDR Discovery Message

If (CDR Response Message(s) received)

Then

Begin

Select CR with Maximum C.

DR sends CDR selection Message to CR

CR changes its state to CDR

CR broadcast Change status Message

End

Else

$\Delta=\Delta+\Omega$.

Until CDRFound $=$ True

$\mathrm{CDRTP}=\mathrm{DRTP}+\Delta$

$\Delta$ - is the transmission power among DR and CDR.

DR informs the selected CDR by including this value in 
CDR Selection Message, this value is very important for CDR. By using the $\Delta$ value. CDR can determine its transmission power that guarantees the connectivity to all domain members. See figure3.

After the selection of DR, IDR, CDR and CR the status of other devices can be simply determined by algorithm5.

\section{Algorithm5. CF Determination \\ //C- Connections number}

If (Device status not determined ) then

If (Device $\mathrm{C}=0$ ) Then Device Status= Unconnected

Else Begin

Device status $=\mathrm{CF}$

Send Device Status Message to Neighbor End.

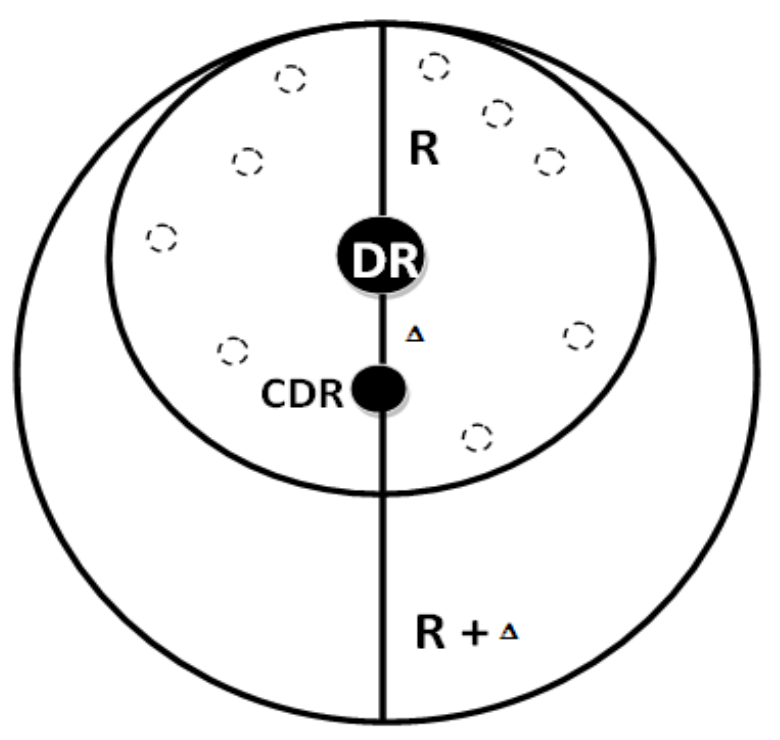

Figure3. CDR Transmission Range.

\section{Routing the Message}

Each member in the domain maintains a list of neighbors addresses s. Each Device in the domain also stores the address of the DR and CDR. DR also maintains members and IDR Addresses. Whenever a device generates a request to transfer the data to a particular device, it checks the destination address in its list. If the matching device is found in the address list, message is transferred to that device. If no match is found, then the Message will be sent to DR. DR will again check for the match in its Address table. If no match is found, DR will forward Message to IDR. This process will continue till the destination device is reached. If Destination device not found or hops exceed maximum acceptable hops count, unreachable error message will be reported. See fig. 4 .

\section{CONCLUSION AND FUTURE WORK}

Dynamic and unpredictable topology, limited bandwidth, limited resources in terms of battery and storing capacity are the major characteristics of MANET. The proposed scheme of domain formation improves many characteristics of MANET. This scheme is applicable and does not depend on a specific architecture or topology of MANET, it doesn't require central device to start domain formation.

The proposed Scheme reduces the storage space requirement of domain members by storing only the addresses of neighbors. Each device does not require maintaining the addresses of all MANET members.

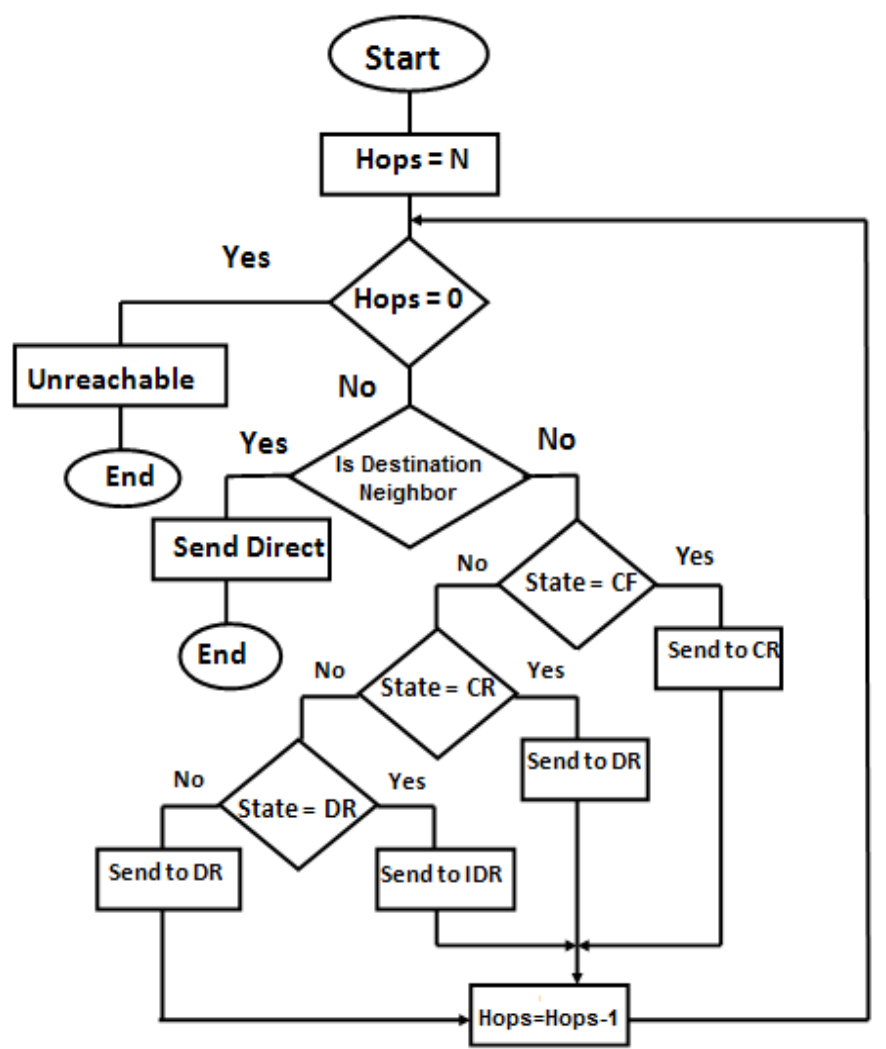

Figure4. Flow chart of the Message Routing.

It reduces broadcasts messages during the message routing or during domain formation which reduces the power consumption and enhances performance.

The proposed scheme is generally scalable and adaptive to mobility due to the fact that each node relies on local information collected from nearby nodes.

The stability achieved by Selecting CDR and by the way the domain formed and the messages forwarded.

Our future work aims to find Security and Maintenance solutions to our proposed Scheme.

\section{REFERENCES}

[1] "Mobile ad-hoc network" fromhttp://en.wikipedia.org/wiki/ Mobile_adhoc_network.

[2] Elizabeth M.Royer and Chai-Keong Toh, "A Review of current routing protocols for Ad-HocMobile Networks", IEEE Personal Communications, Vol. 6, No. 2, pp. 46-55, April 1999.

[3] S.Samanta, S.S.Ray, S.SenGupta, M.K.Naskar, "A Novel Algorithm for Managing Network Configuration", Asian International Mobile Computing Conference 2006, Kolkata, Jan 04-07, pp. 51-58.

[4] S.S.Basu and A.Chaudhari, "Self-adaptive Topology Management for Mobile Ad- hoc Network", IE(I) Journal-ET, Vol.84, July 2003. 
[5] Soumya Sankar Basu, Atal Chaudhari, "Self- Adaptive MANET: A CentralizedApproach", Foundations of Computing and Decision Sciences, Vol.29, 2004

[6] R. Wattenhofer, L. Li, P. Bahl, and Y.-M.Wang, a Distributed Topology Control for Power Efficient Operation in Multihop Wireless Ad Hoc Networks, ${ }^{\circ}$ Proc. Infocom 2001, Apr. 2001.

[7] Errol L. Lloyd, Rui Liu,Madhav V. Marathe Ram Ramanathan S. S. Ravi Algorithmic Aspects of Topology Control Problems for Ad hoc Networks IEEE Mobile Ad Hoc Networking and Computing (MOBIHOC), June 2002.

[8] R. Braden, D. Clark, and S. Shenker, "Integrated services in the Internet architecture: an overview," 1994, IETF RFC 1633.

[9] Yu-Xuan Wang, Forrest Sheng Bao "An Entropy-based Weighted Clustering Algorithm and Its Optimization for Ad hoc Networks" Third IEEE International Conference on Wireless and Mobile Computing, Networking and Communications (WiMob 2007) 0-7695- 2889-9/07 2007

[10] S. Blake, D. Black, M. Carlson, E. Davies, Z.Wang, andW. Weiss, “An architecture for differentiated services," 1998, IETF RFC 2475.

[11] J. Wu and H.L.Li, "On Calculating Connected Dominating Set for Efficient Routing in Adhoc Wireless Networks",Proc 3rd Int'l. Wksp. Discrete Algorithms and Methods for Mobile Comp. and Commun. 1999 pp. $7-14$

[12] Qi Xue , Aura Ganz "Ad hoc QoS on-demand routing (AQOR) in mobile ad hoc networks ". Journal of Parallel and Distributed Computing,(63), 154-165, 2003.

[13] Taewook Kang, Jangkyu Yun, Hoseung Lee,Icksoo Lee,Hyunsook Kim,Byunghwa Lee,Byeongik Lee,Kijun Han "A Clustering Method for Energy Efficient Routing in Wireless Sensor Networks", Proceedings of the 6th WSEAS Int. Conf. on Electronics, Hardware, Wireless and Optical Communications, Corfu Island, Greece, February 16-19, 2007 133.
[14] M. Mirhakkak, N. Schult, D. Thomson, "A new Approach for providing Quality-of-Service in a Dynamic Network Environment", The MITRE Corporation, Network and Communications Engineering Department, Reston, Virginia.

[15] Y.Z.P Chen and A.L Liestman, “Approximating Minimum Size Weakly - Connected Dominating Sets for ClusteringMobile Adhoc Networks", in Proc 3rd ACM Int'l. Symp. Mobile Adhoc Net. \& Comp, June 2002, pp. $165-72$.

[16] Hannan XIAO, Winston K.G. SEAH, Anthony LO and Kee Chiang CHUA “ Flexible Quality Service Model for Ad-HOC Networks". In proceedings of the IEEE Vehicular Technology Conference, Tokio, Japan, May 2000.

[17] Tzay-Farn shih and Hsu-Chun Yen "Core Location- Aided ClusterBased Routing Protocol for Mobile Ad Hoc Networks Proceedings of the 10th WSEAS International Conference on Communications, " Vouliagmeni, Athens, Greece, July 10-12, 2006 (pp223-228).

[18] SWAN Project: http://comet.columbia.edu/swan, 2002.

[19] Vikas Kawadia and P.R.Kumar, "Power Control and Clustering in Adhoc Networks", IEEE Proc. INFOCOMconference 2003, pp 459 469.

[20] P. Basu, N. Khan, and T.D.C. Little, "A Mobility Based Metric for Clustering in Mobile Adhoc Networks", in Proc.IEEE ICDCSW'01, Apr 2001, pp. $413-18$.

\section{AUTHOR PROFILE}

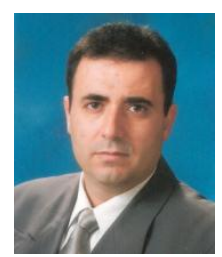

Adwan AbdelFattah is an assistant professor at the Computer Science Department of the Arab American University of Jenin, Palestine. Previously he worked at Philadelphia and Zarka Private University, Jordan. He received his $\mathrm{PhD}$ degree from the National Technical University of Ukraine in 1996. His research interests include Computer Networks, Computer Architecture, Cryptography and Networks Security. 